\title{
On the Use of Fourier Transform Infrared (FT-IR) Spectroscopy and Synthetic Calibration Spectra to Quantify ?1 Gas Concentrations in a Fischer-Tropsch Catalyst System
}

\author{
Frank T. Ferguson, ${ }^{a, \star}$ Natasha M. Johnson, ${ }^{b}$ Joseph A. Nuth III \\ ${ }^{a}$ Catholic University of America, Dept. of Chemistry, Washington, D.C. 20064 USA \\ ${ }^{\mathrm{b}}$ NASA-Goddard Space Flight Center, Astrochemistry Laboratory, Greenbelt, MD 20771 USA
}

\begin{abstract}
One possible origin of prebiotic organic material is that these compounds were formed via Fischer-Tropsch-type (FTT) reactions of carbon monoxide and hydrogen on silicate and oxide grains in the warm, inner-solar nebula. To investigate this possibility, an experimental system has been built in which the catalytic efficiency of different grain-analog materials can be tested. During such runs, the gas phase above these grain analogs is sampled using Fourier transform infrared (FT-IR) spectroscopy. To provide quantitative estimates of the concentration of these gases, a technique in which high-resolution spectra of the gases are calculated using the highresolution transmission molecular absorption (HITRAN) database is used. Next, these spectra are processed via a method that mimics the processes giving rise to the instrumental line shape of the FT-IR spectrometer, including apodization, self-apodization, and broadening due to the finite resolution. The result is a very close match between the measured and computed spectra. This technique was tested using four major gases found in the FTT reactions: carbon monoxide, methane, carbon dioxide, and water. For the ranges typical of the FTT reactions, the carbon monoxide results were found to be accurate to within $5 \%$ and the remaining gases accurate to within $10 \%$. These spectra can then be used to generate synthetic calibration data, allowing the rapid computation of the gas concentrations in the FTT experiments.
\end{abstract}

Index Headings: Fourier transform infrared spectroscopy; FT-IR spectroscopy; Apodization; HITRAN database; Fischer-Tropsch reaction; FTT; Calibration; Quantitative analysis.

\section{INTRODUCTION}

An important question in the origin of life is, what is the source of the necessary prebiotic molecules? In their classic experiment, Miller and Urey ${ }^{1}$ were able to synthesize amino acids under an atmosphere of methane, ammonia, and water with an arc discharge. Unfortunately, the Miller-Urey experiment requires this type of reducing atmosphere and later research seems to suggest that the Earth's primordial atmosphere was only slightly reducing, consisting mostly of nitrogen and carbon monoxide. Other possibilities for the origin of such prebiotic material include submarine vents, but a problem with these vents is that the production rate of organic material is predicted to be too low. Comets have long been proposed as a delivery mechanism of prebiotic material to the Earth. Many volatile organic species have been detected in comets, and the first

Received 12 March 2015; accepted 10 April 2015.

* Author to whom correspondence should be sent. E-mail: frank.t. ferguson@nasa.gov.

DOI: $10.1366 / 15-07950$ detection of an amino acid in a comet was reported in samples taken from comet 81P/Wild 2 during the Stardust mission. ${ }^{2}$

Another possible origin for significant quantities of organic molecules is that they were produced in the warm, inner-solar nebula via a Fischer-Tropsch-type (FTT) catalytic reaction of carbon monoxide and hydrogen on silicate and oxide dust grains. ${ }^{3}$ To investigate this possibility, fine-grained dust analogs of iron or magnesium silicates are produced in an experimental system. In the case of iron silicate grains, the volatile precursor iron pentacarbonyl is reacted at elevated temperatures along with flowing oxygen, hydrogen, and silane mixtures. The grains nucleate and grow in the flowing stream and deposit in a thin layer on a collection plate downstream from the reaction zone. Magnesium silicate grains are produced in a similar manner by vaporizing pure magnesium metal under similar gas streams. These grain analogs are later placed in a catalyst experimental system and heated under various initial atmospheres of carbon monoxide (CO) and hydrogen $\left(\mathrm{H}_{2}\right)$. Organic compounds $\left(\mathrm{C}_{n} \mathrm{H}_{(2 n+2)}\right)$ and water $\left(\mathrm{H}_{2} \mathrm{O}\right)$ are formed via the Fisher-Tropsch reaction:

$$
n \mathrm{CO}+(2 n+1) \mathrm{H} 2 \rightarrow \mathrm{C}_{n} \mathrm{H}_{(2 n+2)}+n \mathrm{H}_{2} \mathrm{O}
$$

In industrial applications, the goal is to maximize the formation of higher-order organics (large $n$ in $\mathrm{C}_{n} \mathrm{H}_{(2 n+2)}$ ) and minimize the production of methane $(n=1)$.

The reaction progress is monitored using Fourier transform infrared (FT-IR) spectroscopy of samples of the gas mixture over time. Once the initial charge of carbon monoxide is depleted (determined using the FTIR spectrometer measurements), these experiments are stopped, the system is refilled with another charge of gas, and the experiments are started again. One interesting observation about these FTT experiments is that the rate of reaction tends to increase as more batches are run; that is, the production of methane occurred faster for later batches than for the first run. ${ }^{4}$ This differs from results using a traditional industrial catalyst, in which the catalyst material becomes poisoned with continued use and eventually loses its effectiveness.

To quantify the reaction rates between dust substrate materials, a method to make rapid quantitative calculations of the gas-phase concentration was developed. In this method, high-resolution transmission spectra of the molecules observed in these FTT reactions are calculated using data from the High-Resolution Transmission 


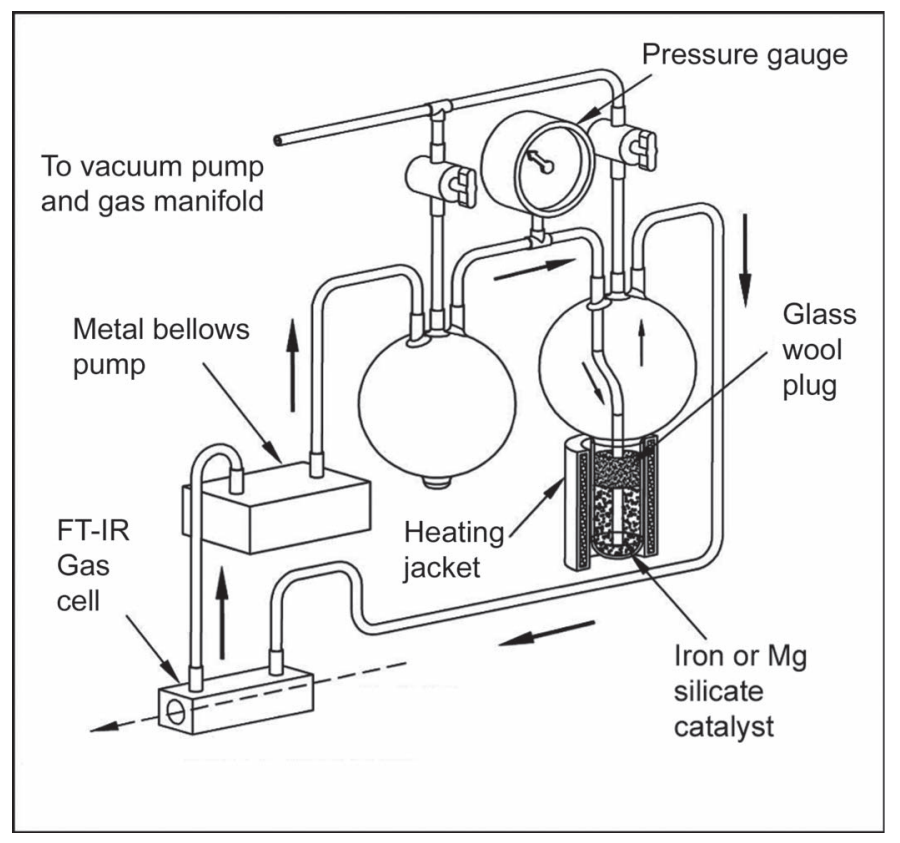

FIG. 1. Diagram of FTT catalyst system.

Molecular Absorption (HITRAN) database..$^{5,6}$ These FTT reactions are well suited for this approach because many of the molecules expected in the FTT reactions are included in this database. The effect of instrumental broadening is applied to these high-resolution calculated spectra and used it to produce synthetic calibration data, enabling the rapid determination of gas concentrations.

\section{CATALYST SYSTEM DESCRIPTION}

As noted in the Introduction, dust grain analog samples were produced in a condensation flow apparatus. After collecting the samples, they were placed in a quartz finger section of a Pyrex reaction vessel, as

?3 shown in Fig. 1. During an experimental run, the system was evacuated and filled with a mixture of gases just below atmospheric pressure (typically 700 Torr). Although the actual gas concentrations varied, a typical run consisted of 75 Torr carbon monoxide, 75 Torr nitrogen, and 550 Torr hydrogen. Filling was done using a manifold connected to the various gases, and the gas pressures were measured using a $390 \mathrm{HA}-01000$ pressure gauge (MKS). Once filled with gases, the system was isolated from the vacuum pump-gas manifold and heat was applied to the sample using a heating mantle. The temperature of the mantle was monitored using a type $\mathrm{K}$ thermocouple and was controlled using a digital

?4 temperature controller. The reactant gases were circu-

?5 lated using a metal bellows pump. This pump was chosen to minimize exposure to possible hydrocarbon contaminants and served two purposes. First, the flow fluidized the fine dust grains in the heated quartz finger, promoting the catalytic reaction. Second, it transported the gases through a length of tubing to an FT-IR

?6 spectrometer absorption cell. During the experiments, the spectra were taken at timed intervals to monitor the gas concentrations.
A variety of complex organics may be produced on the catalysts; fortunately, the major, infrared-absorbing gaseous species involved in the FTT reaction (carbon monoxide, carbon dioxide, methane, and water) are included in the HITRAN database. Also, while the catalysts were heated to temperatures ranging from 200 to $600{ }^{\circ} \mathrm{C}$, the gases were being recirculated through long tubes to the FT-IR spectrometer cell. Therefore, the absorption measurements were made at room temperature, also simplifying the analysis of the measured spectra.

\section{QUANTITATIVE FOURIER TRANSFORM INFRARED SPECTROSCOPY MEASUREMENTS}

Although FT-IR spectrometry is a common laboratory diagnostic tool, quantitative measurements using it are difficult because the absorption coefficients depend nonlinearly on temperature, pressure, and concentration. To circumvent this difficulty, calibrations between known amounts of a species and the FT-IR spectrometer measurements can be performed. One drawback of this approach is that, if new experimental ranges are needed, additional calibrations must be made. In some cases, the species may not be stable under the calibration conditions or the calibrations simply may not be feasible.

An absorption line is typically broadened by both Doppler and pressure broadening. In addition, there is an instrument line shape (ILS) of the spectrometer, a broadening that occurs due to the apodization and finite resolution of the instrument. In an FT-IR spectrometer, the effect of finite resolution is mathematically equivalent to multiplying a high-resolution interferogram by a rectangular function that is one over the resolution range and zero at all higher resolutions. Such a truncation of the interferogram can produce unwanted oscillations in the spectra when it is transformed back to the frequency domain, and these oscillations may be mistaken for actual absorption features. As a result, the interferogram is often multiplied by an apodization function that reduces these oscillations. In addition to the applied apodization function, there is further broadening of the spectrum due to the field of view of the spectrometer. Because the infrared source is not a point source and has a finite size, light rays emanate from the source at different angles and travel through the beamsplitter and mirrors with different optical path lengths. The result is an additional instrumental broadening, termed self-apodization.

The effect of this ILS can be simulated by convolving a computed spectrum with the Fourier transform of the instrument apodization function and the self-apodization term. The ILS for an FT-IR spectrometer is typically infinite in extent, making the technique computationally expensive. Further, the technique is usually reserved for isolated lines because neighboring peaks would be subject to the same ILS calculations, adding to the complexity of the computation.

Another technique, first demonstrated by Griffith $^{7}$ and Morrison and Taweechokesupsin, ${ }^{8}$ simulates the mathematical processes occurring in a FT-IR spectrometer. First, a high-resolution spectrum over the desired range

Volume 69, Number 11, 2015 
is calculated. This high-resolution spectrum in the wavenumber domain is then inverse Fourier transformed to the retardation domain to give a highresolution interferogram. Apodization is applied to this interferogram, and the interferogram is truncated to match the resolution of the FT-IR spectrometer. The interferogram is then Fourier transformed back to the frequency domain to produce the final processed spectrum.

Griffith $^{7}$ outlined this technique and demonstrated its use using three different cases and instrument resolutions: (1) a closed absorption cell; (2) a long-path, open FT-IR spectrometry; and (3) solar FT-IR spectroscopy. Agreement between the measured and calculated spectra was excellent. Griffith noted that this technique offers several nice advantages, including speed and convenience, handling situations in which calibrations may not be possible, and handling Beer-Lambert nonlinearities.

Morrison and Taweechokesupsin ${ }^{8}$ demonstrated this technique using a detailed example of a single, isolated methane line with the goal of using the technique to analyze FT-IR spectrometer measurements in a vapordeposition reactor. Morrison and Taweechokesupsin again noted that this technique is useful in situations in which species are not suitable for calibrations or wide ranges in temperature or pressure are encountered. They also noted the technique is particularly suitable for non-isolated lines and easily handles bands of overlapping features. Similar to Griffith, Morrison and Taweechokesupsin found excellent agreement between the measured and calculated spectra for methane with background gases of air and hydrogen.

Once synthetic spectra are available, the next step is to use these data to predict concentrations. One of the most commonly used techniques is the classical least squares (CLS) method. ${ }^{9}$ The CLS method is a multivariate technique in which a set of calibrations is made, using either experiments or synthetic spectra, bracketing the expected experimental range. Predictions are made based on the Beer-Lambert law and these calibration data by minimizing the error between the measured and predicted spectra. An advantage of this technique is that it does not require spectrally isolated components, although it does require calibration data for every component in the mixture. Having unknown components in the mixture spectra will result in prediction errors.

There are several examples of the use of synthetic spectra and the CLS method in the literature. Cant et al. ${ }^{10}$ used an FT-IR spectrometer to sample gas flow through a catalytic reactor, studying nitrous oxide formation in exhaust streams over rhodium, platinum, and palladium catalysts. Synthetic spectra have been generated from the HITRAN database for several reactant species and the concentrations determined via least squares. Flores et al. ${ }^{11}$ similarly used synthetic spectra and CLS to determine nitrous oxide and nitric acid concentrations. Flores et al. also noted that using synthetic spectra was particularly advantageous in this case because no reference standards of nitric acid were available. Yokelson et al. ${ }^{12}$ were able to retrieve concentrations for the smoldering combustion of a biomass using an open-path Fourier transform infrared (OP-FT-IR) spec- trometer system and synthetic spectra, combined with CLS. Cowan et al. ${ }^{13}$ studied the catalytic reduction of nitromethane in a flow system. The progress of the reaction was monitored using a FT-IR spectrometer and concentrations were determined using CLS and a combination of experimental calibrations and spectra generated from the HITRAN database.

Smith et al. ${ }^{14}$ noted that at high concentrations there can be differences between the CLS predictions and actual concentrations due to Beer-Lambert nonlinearities. Using a nonlinear least squares (NLLS) fitting procedure, they were able to demonstrate a better than $5 \%$ agreement for measurements using an OP-FT-IR spectrometer for the three most abundant greenhouse gases in the atmosphere-carbon monoxide, carbon dioxide, and methane-for a wide range of path lengths. This NLLS technique combined with synthetic spectra was also used by Burling et al. ${ }^{15}$ to study the trace gas emissions from a biomass burning using an OP-FT-IR spectrometer and by Cant et al. $^{16}$, in a study of the catalytic reduction of nitrous oxide by carbon monoxide over supported platinum catalysts.

Fleckl et al. ${ }^{17}$ focused on in situ measurements in a combustion chamber at elevated temperature. They calculated synthetic spectra using the technique developed by Griffith $^{7}$ and by Morrison and Taweechokesupsin ${ }^{8}$ to account for the FT-IR spectrometer ILS, including self-apodization. In general, they found extremely good agreement for carbon monoxide, carbon dioxide, and water with temperatures up to $1100^{\circ} \mathrm{C}$, but they did note some deviations for carbon dioxide at these elevated temperatures.

Sepman et al. ${ }^{18}$ also made quantitative measurements, using a FT-IR spectrometer to study the pyrolysis process of a biomass. In contrast, they did not employ the technique used by Griffith ${ }^{7}$ and by Morrison and Taweechokesupsin ${ }^{8}$ but, rather, looked at the integral absorptivity (equivalent width) over features. Over proper integration boundaries, the equivalent width is independent of the apparatus function of the FT-IR spectrometer. Choosing the integration limits requires some careful considerations; this choice is a balance between increasing the range to include the instrument function and absorption, on the one hand, and the desire to avoid possible neighboring features, on the other. To explore this, Sepman et al. calculated spectra and simulated the effect of different ILSs via convolution with the calculated spectra. These authors obtained very good results for ammonia, carbon monoxide, ethylene, ethane, methane, and nitric oxide.

In this study, synthetic spectra were generated using the HITRAN database, and the ILS of the FT-IR spectrometer was simulated using the technique of Griffith $^{7}$ and of Morrison and Taweechokesupsin. ${ }^{8}$ Concentrations were found by comparing the integrated absorptivity of spectra over a particular wavelength range to those taken from synthetic calibration spectra. Although spectrum-fitting methods such as CLS could offer more accurate measurements, the simpler technique of using the integrated absorptivity resulted in sufficient accuracy over a wide range of experimental concentrations. In addition, because the total system pressure changes in the FTT system as the reaction 
proceeds, spectrum-fitting techniques would require a large number of calibration spectra. This technique is well suited for the gas concentrations expected in this study because, at the concentrations of a typical FTT run, many overlapping lines and integrations over these

?7 bands would be more useful. Because the measured and calculated spectra are a close match, this softens some of the restrictions on choosing appropriate integration ranges. Finally, computing the concentrations from integrated absorptivity measurements can be done very rapidly, and the technique can be used to monitor the experiments in real time.

\section{HIGH-RESOLUTION SPECTRA BASED ON THE HIGH-RESOLUTION TRANSMISSION MOLECULAR ABSORPTION DATABASE}

The HITRAN database ${ }^{5}$ is a spectroscopic parameter compilation that can be used to calculate high-resolution, line-by-line transmission spectra. ${ }^{6}$ The most recent version was just recently released and includes data for 47 molecules. In this section, a brief description of how high-resolution spectra are calculated from this database is given.

The transmission $\tau_{i}(v)$, at a particular spectral frequency $v$ for a single absorption line $i$, is given by

$$
\tau_{i}(v)=\frac{I}{I_{0}}=e^{-\left(k_{i}(v) n L\right)}
$$

where $I$ is intensity of the light after absorption, $I_{0}$ is the intensity of the original source, $k_{i}(v)$ is the absorption coefficient of the line, $n$ is the molar concentration of the absorbers, and $L$ is the path length of the sample. The transmission is measured by recording the ratio of the intensity of the light after absorption, I, to the intensity of the original source, $I_{0}$. The absorption coefficient for a line is the product of the spectrally independent line strength, $S_{i}(T)$, and the line-shape function, $f\left(v-v_{c, i}\right)$ :

$$
k_{i}(v)=S_{i}(T) f\left(v-v_{\mathrm{c}, i}\right)
$$

?8 where $v_{\mathrm{c}, i}$ is the frequency of the line center. The line strength $S_{i}(T)$ is a function of the temperature, $T$, and the values for the line strengths at $296 \mathrm{~K}$ are given in the HITRAN database. For other temperatures, the line strengths are calculated using:

$$
S_{i}(T)=S_{i}\left(T_{0}\right) \frac{\exp \left(\frac{-h c E_{\mathrm{L}, i}}{k_{\mathrm{B}} T}\right) Q_{\mathrm{T}}\left(T_{0}\right)\left[1-\exp \left(\frac{-h c v_{\mathrm{c}, i}}{k_{\mathrm{B}} T}\right)\right]}{\exp \left(\frac{-h E_{\mathrm{L}, i}}{k_{\mathrm{B}} T_{0}}\right) Q_{\top}(T)\left[1-\exp \left(\frac{-h c v_{\mathrm{c}, i}}{k_{\mathrm{B}} T_{0}}\right)\right]}
$$

where $S_{i}\left(T_{0}\right)$, is the line strength at a reference temperature, $T_{0}$ (296 $\mathrm{K}$ for the HITRAN database), $h$ is Planck's constant, $c$ is the speed of light, $k_{B}$ is Boltzmann's constant, and $v_{c, i}$ is the transition frequency

?9 for the particular line, $i$. The term $E_{\mathrm{L}, i}$, is the lower-state energy of the transition, and $Q_{\mathrm{T}}$ is the total internal partition function. The value of the lower-state energy is available in the HITRAN database, and tabulated values for the total internal partition function are also available in HITRAN from 70 to $3000 \mathrm{~K}$.

The line-shape function gives the spectral distribution of the absorbed intensity relative to the line center. The frequency of this line center, $v_{\mathrm{c}, i}^{0}$, at a reference pressure, $P_{0}$, of $1 \mathrm{~atm}$ is given in the HITRAN database. For other pressures, this line center, $v_{\mathrm{c}, i}$, is given by:

$$
\mathrm{v}_{\mathrm{c}, i}=\mathrm{v}_{\mathrm{c}, i}^{0}+\partial\left(\frac{P}{P_{0}}\right)
$$

where $\delta$ is the air-broadened pressure shift of the line transition, which is also given in the HITRAN database.

The two predominant line-broadening mechanisms are pressure and Doppler broadening. As the absorbing gas undergoes thermal motion, the distribution of velocities broadens the line shape. This broadening is quantified by the Doppler half-width, $\alpha_{D, i}$, given by

$$
\alpha_{\mathrm{D}, i}=\frac{v_{\mathrm{c}, i}}{c} \sqrt{\frac{2 k_{\mathrm{B}} T}{m}}
$$

where $m$ is the molecular mass of the absorbing species. Doppler broadening is typically predominant at low pressures and results in a Gaussian line shape.

At higher pressures, the line is broadened by collisions, resulting in a Lorentzian line shape. These collisions may be between like molecules or foreign species, depending on the relative amounts of each species. The HITRAN database contains information on the self-broadening half-width, $\alpha_{\mathrm{Ls}, i}^{0}$, and the air-broadened half-width, $\alpha_{\mathrm{La}, i}^{0}$. For a mixture, these values are weighted by the mole fraction of the absorbing species, $x_{\mathrm{a}}$, to give the Lorentzian half-width at the HITRAN reference temperature and pressure:

$$
\alpha_{\mathrm{L}, i}^{0}=\left(1-x_{\mathrm{a}}\right) \alpha_{\mathrm{La}, i}^{0}+x_{\mathrm{a}} \alpha_{\mathrm{Ls}, i}^{0}
$$

The Lorentzian half-width is adjusted to different temperatures and pressures using:

$$
\alpha_{\mathrm{L}, i}=\alpha_{\mathrm{L}, i}^{0}\left(\frac{P}{P_{0}}\right)\left(\frac{T_{0}}{T}\right)^{\gamma}
$$

where $\gamma$ is the temperature-dependence exponent, also given in the database.

For conditions in which both Doppler and collisional broadening are significant, the Gaussian and Lorentzian line shapes are combined using the Voigt line shape:

$$
f\left(v-v_{\mathrm{c}, i}\right)=\frac{y_{i}}{\alpha_{D, i} \pi^{3 / 2}} \int_{-\infty}^{+\infty} \frac{\exp \left(-t^{2}\right)}{y_{i}^{2}+\left(x_{i}-t\right)^{2}} d t
$$

where

$$
\begin{aligned}
& y_{i}=\frac{\alpha_{\mathrm{L}, i}}{\alpha_{\mathrm{D}, i}} \\
& x_{i}=\frac{v-v_{\mathrm{c}, i}}{\alpha_{\mathrm{D}, i}}
\end{aligned}
$$

and $t$ is an integration variable.

For a band of lines, the total transmission, $\tau(v)$, is given by

$$
\tau(v)=\exp \left(-\sum_{i=1}^{n_{1}}\left(k_{i}(v) n L\right)\right)
$$


where Eq. 12 is a sum over $n_{1}$ multiple lines that can overlap and contribute to absorption at a particular frequency. In a calculation of a band of lines, the neighboring lines outside the limits of the band must also, of course, be included in the calculation. The resulting profile is the resulting transmission profile if it were measured using an instrument of very high resolution.

\section{CALCULATION OF INSTRUMENTAL BROADENING}

As noted earlier in this work, the measured transmission, $\tau_{\mathrm{m}}$, is the convolution of the true transmission

$$
\tau_{\mathrm{m}}(v)=\int_{0}^{\infty} \tau(v) \operatorname{ILS}\left(v-v_{0}\right) d v
$$

If the ILS function is known, the measured transmission spectrum can be calculated from Eq. 13, but it is computationally expensive because the ILS function extends quite far.

A second approach is to perform mathematical operations analogous to those that occur in the FT-IR spectrometer. Morrison and Taweechokesupsin ${ }^{8}$ gave a detailed example of this process for a single methane absorption line. In this article, a detailed example with a band of carbon monoxide absorption lines over a finite range is provided.

The resolution of the FT-IR spectrometer is dependent on the mirror retardation, and to achieve infinite resolution, the mirror would have to be moved from zero to infinity. In actuality, the mirror is moved a finite distance, which sets the resolution. This is mathematically equivalent to truncating a very-high-resolution spectrum at all points except for the finite distance that the mirror actually moves. To simulate the instrumental effects on the measured spectra, this process is mimicked. A high-resolution spectrum is initially calculated, and the spectrum is truncated and apodized, just as in a real FT-IR spectrometer.

As noted by Morrison and Taweechokesupsin, ${ }^{8}$ there are two restrictions on the calculation of the initial highresolution transmission spectrum. First, the resolution must be high enough to fully resolve the lines in the spectrum (i.e., four to five points per line). Second, the number of points must be a power of two so the fast Fourier transform (FFT) algorithm can be applied. The data can, of course, be zero-padded up to the next power of two to allow work with smaller calculated ranges. In this study, the high-resolution spectra were calculated at $(1 / 256) \mathrm{cm}^{-1}$ intervals.

An overview of this technique is given in Fig. 2. In this example, the transmission spectrum for 100 Torr carbon monoxide at $296 \mathrm{~K}$ over a path length of $10.5 \mathrm{~cm}$ was calculated from the HITRAN database using the method outlined earlier. Data were calculated from 2145 to $2161 \mathrm{~cm}^{-1}$ with a point-to-point spacing of $(1 / 256) \mathrm{cm}^{-1}$; this spectrum is shown as the unshaded section of Fig. $2 a$. To produce the high-resolution interferogram, these data must be inverse Fourier transformed. Also, to keep the interferogram purely real with no imaginary compo-

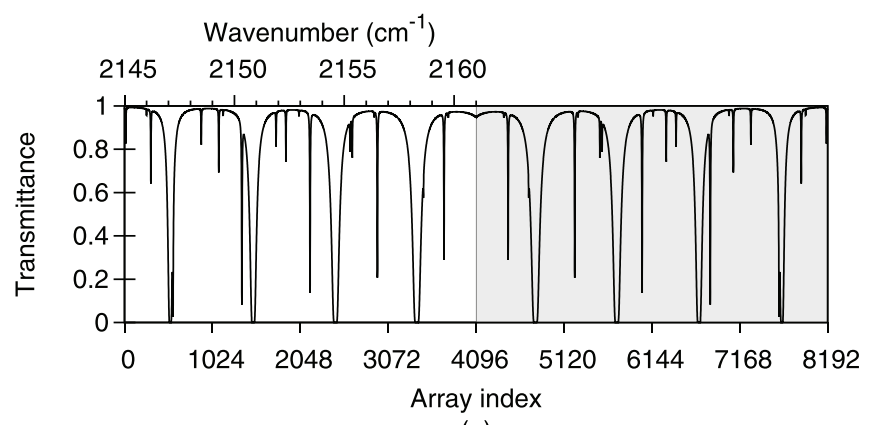

(a)

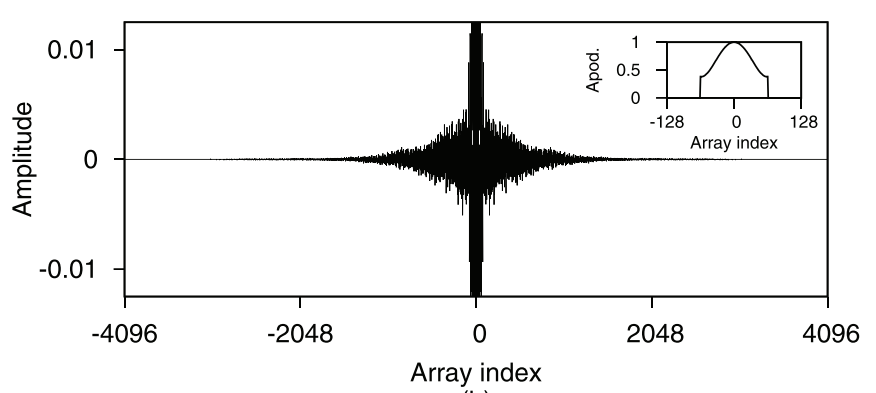

(b)

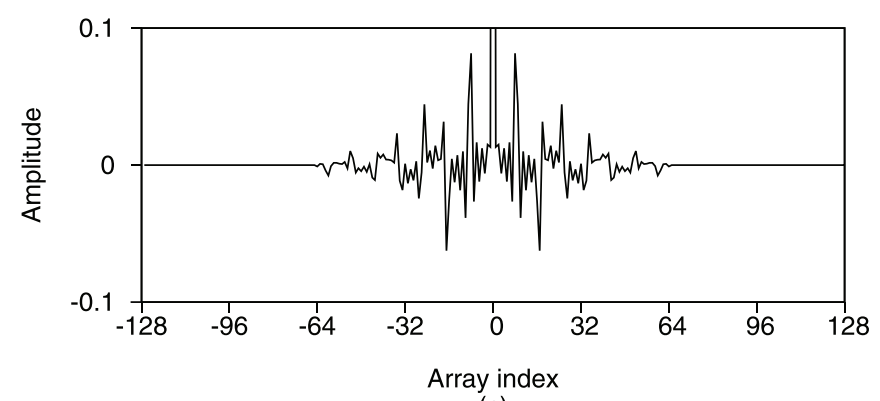

(c)

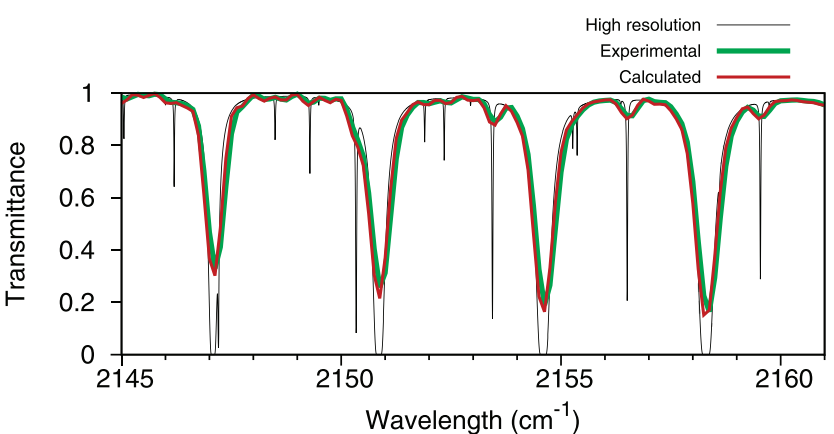

(d)

FIG. 2. Overview of steps to account for instrumental broadening. (a) The original, high-resolution spectrum is mirrored. (b) The mirrored high-resolution spectrum is then inverse-Fourier-transformed to give a high-resolution interferogram. Inset: The apodizing function. (c) The high-resolution interferogram is then truncated, and apodization is applied. (d) The high-resolution, truncated, and apodized interferogram is then Fourier-transformed to yield the final spectrum; for comparison, the original high-resolution spectrum is also shown (thin black line).

nents, the original data must be even. Therefore, the calculated spectrum is mirrored, as shown by the shaded section of Fig. 2a. The inverse Fourier transform of the spectrum shown in Fig. $2 \mathrm{a}$ is shown in Fig. 2b. 
We highlight a couple of computational notes regarding this calculation. Data passed to an FFT algorithm run from 0 to $N-1$ (where $N$ is a power of two). To keep the function even for the FFT algorithm, $X(n)=X(N-n)$. In this particular example, values from 0 to 8191 were passed to the FFT routine. Therefore, 4097 values were needed for the original computed spectrum. Next, the interferograms shown in Fig. 2 have been reordered to place the zero-frequency, or centerburst, value at the center. This is different from the way the data are returned from a typical FFT algorithm. Typically, when data are returned from a FFT routine, the zero-frequency component is the first value in the array, followed by the positive frequency values and, then, the negative frequency values. For clarity in viewing the operations to the interferogram, the interferogram is reordered so that the centerburst is given by index 0 and placed in the center of the figure.

Figure $2 \mathrm{~b}$ is similar to an interferogram taken at a high resolution. In this study, the weak Norton-Beer (WNB) apodization function ${ }^{19}$ was used for all scans; the form of this apodizing function is shown in the inset of Fig. $2 b$. The resolution of all the experimental scans taken in this study was $0.5 \mathrm{~cm}^{-1}$. Therefore, by the Nyquist criteria, the sampling of the data must be at $0.25 \mathrm{~cm}^{-1}$ intervals. Using these values, the high-resolution spectrum at sample intervals of $(1 / 256) \mathrm{cm}^{-1}$ must be truncated by a factor of $256 / 4=64$ to give the same effective resolution as the FT-IR spectrometer. Therefore, the original interferogram, shown ranging from an index of -4095 to 4096 , must be multiplied by the apodizing function, which has values ranging from -63 to 64 , as shown in the inset to Fig. $2 b$. When a spectrum is collected, values are actually given at data intervals of $0.125 \mathrm{~cm}^{-1}$. To allow a direct comparison between these values and the computed spectrum, the interferogram is zero-padded up to a range of -127 to 128 , giving interpolated values, just as is done in the FT-IR instrument. Figure $2 c$ is the resulting interferogram after truncation and zero padding have been applied to the original high-resolution interferogram.

As noted earlier, in addition to the selected apodization function there is a self-apodization term related to the finite field of view of the instrument. If the light originated from a point source, then there would only be a single optical path difference. With an extended source, there is, instead, a range of optical path differences that is dependent on the beam divergence. With this extended source, the spectrum is convolved using a boxcar function of width $v \alpha^{2} / 2$ for each wavenumber, where $\alpha$ is the beam divergence halfangle. This effect can be simulated by multiplying the interferogram by $\operatorname{sinc}\left(\pi v \alpha^{2} x / 2\right)$, the Fourier transform of this boxcar function, where $x$ is the optical path difference. The beam divergence is related to measurable quantities, $\alpha=d /(2 F)$, where $d$ is the collimator aperture and $F$ is the focal length of the instrument. Griffith $^{7}$ was able to account for this self-apodization directly based on these known quantities. Another method to account for this self-apodization in an approximate way, used by Morrison and Taweechokesupsin ${ }^{8}$ and Fleckl et al., ${ }^{17}$ is to adjust the applied apodizing function slightly and match the results to spectra measured using the FT-IR spectrometer. In this study, the self-apodization was calculated directly from the focal length and aperture of the spectrometer.

After the truncation, apodization, and zero-padding operations are applied to the high-resolution interferogram, the final computed spectrum is found by Fouriertransforming the data to the frequency domain, as shown in Fig. 2d. Also shown in Fig. 2d is the spectrum for carbon monoxide collected under the same conditions as the calculation. As shown in the figure, the match between the two is very good.

\section{OVERVIEW OF EXPERIMENTAL VALIDATION}

In this section, a comparison between the FT-IR spectra from defined gas mixtures is quantitatively compared to the results from the technique outlined in the earlier sections. These comparisons are made to validate the approach and assess the possible uncertainties in the method. A brief overview of some of the information for these validation experiments is provided in this section.

The predominant infrared-absorbing gases in the FTT reactions are carbon monoxide, methane, carbon dioxide, and water. With the exception of water (discussed later in this work), all gas mixtures were prepared using manometric mixing as measured using a 390HA-01000 pressure gauge and the vacuum-gas manifold system (MKS). This gauge measures pressures in the 0 1000 Torr range with a stated accuracy of $0.005 \%$ of the reading. The gases used as the initial feedstock in the FTT reactions (namely carbon monoxide and hydrogen; Air Products) were of ultra-high purity grade, and these same gases were also used for these comparison experiments. As for the FTT products, a bottle of scientific-grade methane (MG Industries) and a standard bottle of carbon dioxide (99.5\% purity; Air Products) were used for these validation experiments. All the gas mixtures were introduced into the same full experimental system as used for the FTT experiments, and after an initial fill, the gases were recirculated throughout the system using the bellows pump to ensure even mixing. Spectra of the gas mixtures were taken using a drynitrogen-purged Spectrum 100 FT-IR spectrometer (Perkin Elmer), in which the cell (with the exception of the water measurements) was held at room temperature. Each spectrum, including the background scans, was taken from 16 co-added scans. This number of scans is the same as that used during the FTT experiments and is a compromise between noise reduction and sample time. The resolution of all the scans was $0.5 \mathrm{~cm}^{-1}$, and WNB apodization was used. The choice of WNB apodization was made based on the work of den Blanken, ${ }^{20}$ which noted that WNB apodization is a good choice if either high resolution or good quantitative accuracy is required.

There is one additional note regarding the focus of this study. Our quantitative results are from comparing the integral absorptivity over a range of an experimentally measured spectrum to a simulated spectrum calculated using the HITRAN database. Although a large range of concentrations may be calculated using multiple ranges (strong lines for low concentration and weak lines for

Volume 69, Number 11, 2015 
higher concentrations), the goal of this study was to find a simple and rapid method of quantifying gas concentrations relevant to comparing reaction rates or monitoring the progress of the FTT reaction ( $\sim 0.5$ Torr to tens of Torr). Therefore, only a single integration range was used at that time, which appropriately encompasses the expected partial-pressure ranges of the FTT experiments.

Carbon Monoxide Results. Monitoring the amount of carbon monoxide is most important in these FTT catalytic studies. It is the primary feedstock to the reaction, and it is used to determine when the run will be terminated. Typically, FTT runs are made in batches, and the run is considered complete when the amount of carbon monoxide drops to approximately $10 \%$ of its initial level. In this study, the integrated absorptivity over a specified range of the carbon monoxide band is used to determine the concentration. Note regarding the choice of this range that, first, the range of lines should be chosen so that the lines are isolated from the lines of other possible species. Second, the focus of this work was in the prediction of the partial pressures of the reaction gases in the range of a fraction of a torr to tens of torr rather than the detection of small amounts of these species. The spectra calculated using the HITRAN database can be useful in determining appropriate ranges based on the expected amounts of these species. Finally, unlike the equivalent width method, in this method there is some freedom in choosing the integration range. For the equivalent width method, an entire line or group of lines must be chosen and the integration limits must be chosen to entirely bracket the feature but not include neighboring lines. Because the ILS is also included in the calculation, the resulting calculated line shape should be a very close match to the experimentally measured spectrum.

All information needed to calculate the spectrum for carbon monoxide is available except for the foreign-gas broadening information. The predominant background gas in the FTT reactions is hydrogen, and the total pressure typically runs from 700 to 750 Torr. The HITRAN database has only self-broadening and air-broadening coefficient data. In many cases, if a calculation for a gas other than air is needed, it is simply assumed that the air-broadened coefficient is adequate. For example, Morrison and Taweechokesupsin ${ }^{8}$ noted that there was negligible difference between the measured and calculated data for $0.37 \%$ methane in hydrogen at 100 Torr.

Comparisons between the experimentally measured spectra of carbon monoxide in air and the calculations were made, and the agreement between the two was extremely good. Similar runs were later made with hydrogen as the background gas. Again, there was a very good visual match between the computed and measured spectra. A problem arose when the calculations were used to match the measured spectra and determine the gas partial pressure. At the highest concentration of carbon monoxide, approximately 75 Torr, in an atmosphere of hydrogen at approximately 750 Torr total pressure, the calculations predicted concentrations of carbon monoxide approximately $10 \%$ higher than the true values. In contrast, there were excellent matches between the measured and calculated values for pure carbon monoxide and for carbon monoxide in hydrogen at low pressures. It was determined that this over-prediction came from using the airbroadening coefficient.

Fortunately, data on the broadening of this band in hydrogen were available. Régalia-Jarlot et al. ${ }^{21}$ provided data for this band for carbon monoxide $\left({ }^{12} \mathrm{C}^{16} \mathrm{O}\right)$, and the HITRAN air-broadened values were replaced with these data. The values were for only the main isotope, ${ }^{12} \mathrm{C}^{16} \mathrm{O}$, but because this isotope represents $>98 \%$ of the total carbon monoxide concentration, replacing these data was a very effective substitution.

Figure 3 is a comparison between the measured and computed spectra for carbon monoxide over the range 2080-2205 $\mathrm{cm}^{-1}$. Figure $3 \mathrm{a}$ is a plot of 75.2 Torr carbon monoxide at 749.9 Torr total pressure, Fig. $3 \mathrm{~b}$ is a plot 7.65 Torr carbon monoxide at 747.6 Torr total pressure, and Fig. $3 \mathrm{c}$ is a plot of 0.775 Torr carbon monoxide at 750.1 Torr total pressure. As shown in the figures, there is a very close match between the calculated and measured spectra. Because of this close agreement, a plot of the residuals, the measured values minus the calculated values, is shown below each pair of spectra in Figs. 3a-3c.

The match between the calculated and measured spectra is very good; however, the goal of this study was to find a method of computing the gas partial pressure given an experimentally measured spectrum. The cell path length, cell temperature, total pressure, and other necessary parameters were all known. The integrated absorptivity of the spectrum over the given window is computed and the partial pressure of the carbon monoxide matching this area is found by bisection. To test the accuracy of this method, a number of runs were made to compare the measured and computed values (see Table I). Listed in the table are (1) the scan number, the total pressure, cell temperature, partial pressure, and mole fraction of carbon monoxide as determined from mixing and pressure-gauge measurements, (2) the same values computed from the FT-IR spectrometer scans and the HITRAN database, and finally (3) the percentage difference between these two sets of values.

These mixtures were made by adding an initial amount of carbon monoxide and reducing the total pressure using the vacuum system or adding additional background hydrogen gas. The stated accuracy of the pressure gauge is $0.005 \%$ of the reading, and the noted uncertainty in the manometric values is based on this uncertainty carried through the reductions in pressure or additions of hydrogen gas.

To estimate the uncertainty in the spectral measurements, several sources were considered: the uncertainty in the HITRAN line strengths, the uncertainty in the hydrogen gas broadening, and the noise level in the measured spectra. The typical stated uncertainty for the carbon monoxide lines in the range used here is $2-5 \%$. To estimate the effect of such an uncertainty on the results, the results were recalculated using line strengths that were $3.5 \%$ higher. Régalia-Jarlot et al. ${ }^{21}$ estimated the uncertainty on the hydrogen broadening coefficients as better than $5 \%$. Therefore, similar to the line strength calculation, the results were recomputed using the foreign gas broadening coefficients that were 


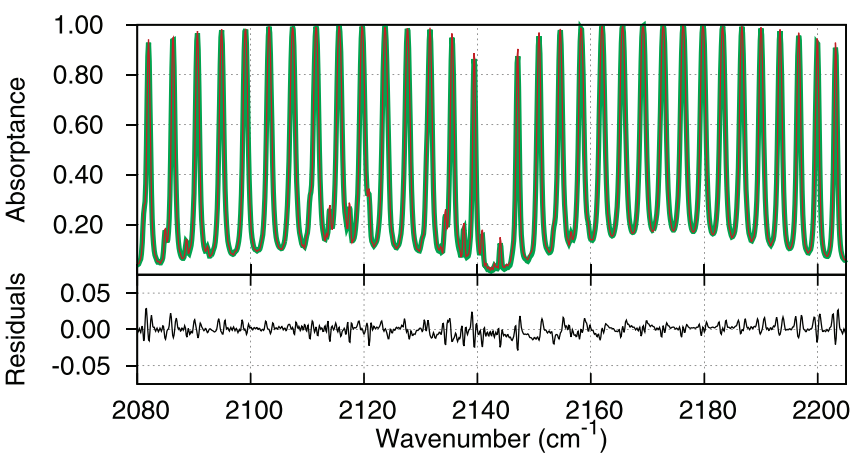

(a)

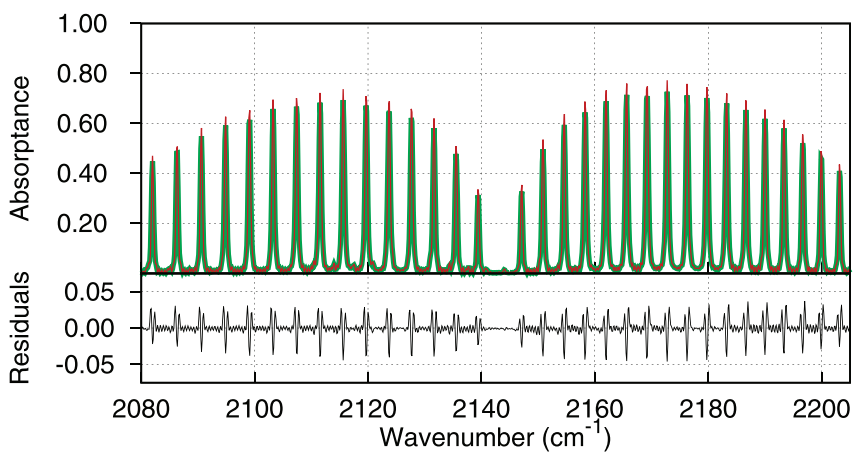

(b)

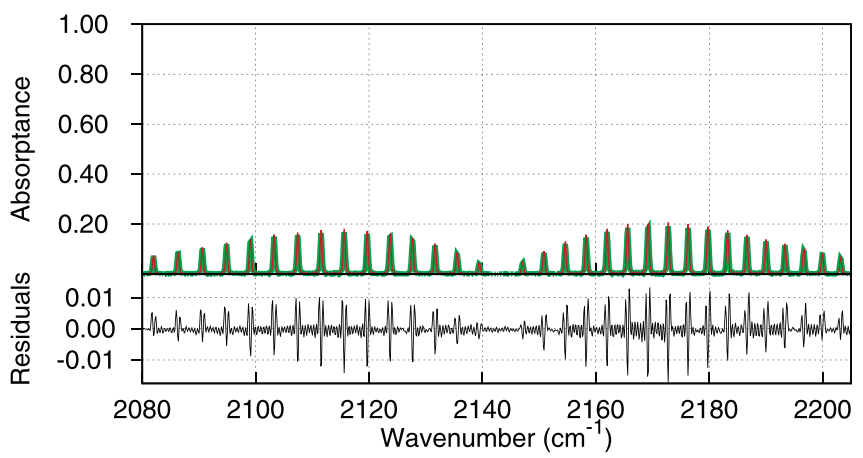

(c)

Experimental Calculated

FIG. 3. Comparison between the measured and calculated spectra for carbon monoxide (CO) in hydrogen at $296 \mathrm{~K}$ and plots of the residuals between the measured and calculated spectra. (a) For 75.2 Torr $\mathrm{CO}$ at 749.9 Torr total pressure. (b) For 7.65 Torr CO at 747.6 Torr total pressure. (c) For 0.775 Torr $\mathrm{CO}$ at 750.1 Torr total pressure.

$5 \%$ higher. Finally, at low signal levels, the noise level in the measured spectra can have a strong influence on the accuracy of the results. To quantify this, several experimental spectra with no absorption were measured and the root mean square (RMS) area of this spectral window was computed. It is common to estimate the detection limits to the concentration as three times this value. In this case, three times the RMS value was subtracted from the experimentally measured area for the scan and again recomputed the results.

The fractional uncertainties from each of these were combined using the root sum of squares to assign a total uncertainty to the spectral results. These values are given as the uncertainty levels of the computed carbon monoxide partial pressures and mole fractions given in the spectral columns (columns 6 and 7 ) of Table I. At the highest partial pressures of carbon monoxide, the largest uncertainty is due to the uncertainty in the foreign gas broadening. As the pressure is reduced and the spectral signal diminishes, the uncertainty due to the noise in the measured spectra becomes dominant. All the spectral results appear to match the manometric results within the stated uncertainties. In general, the agreement between the manometric and spectrally computed values are very good. In fact, for values down to $\sim 0.1$ Torr carbon monoxide, the two agree to approximately $2 \%$, on average.

Methane Results. During the FTT reaction, a significant amount of methane gas is produced; after carbon monoxide, it is the second most abundant, infraredabsorbing species in a catalytic run. At much smaller concentrations, higher-order alkanes are also produced during this FTT reaction. One difficulty in quantifying the amount of methane is that the main band at $3014 \mathrm{~cm}^{-1}$ is the location of the $\mathrm{C}-\mathrm{H}$ stretching vibration, so there is a strong potential for interfering absorption features from these higher-order alkanes. ${ }^{22}$ This required looking at the absorption features of such species, as well as interfering lines from water, and choosing a region of lines isolated from such influence. In the end, a region of weak lines in the range $3100-3152 \mathrm{~cm}^{-1}$ was chosen.

A comparison of the measured and calculated spectra for methane, similar to the procedure used for carbon monoxide, is shown in Fig. 4. Shown are the spectra for 50.1 Torr methane at 749.5 Torr total pressure (Fig. 4a), 4.99 Torr methane at 749.8 Torr total pressure (Fig. 4b), and 0.50 Torr methane at 750.8 Torr total pressure (Fig. 4c). Similar to carbon monoxide, a plot of the residuals for each case is given below each plot of the spectra. In contrast to the carbon monoxide comparison, no attempt was made to correct for the foreign gas broadening and the air-broadened coefficients from the HITRAN database were used. In spite of this, the agreement between the measured and calculated spectra is very good.

The same type of uncertainty estimate was made with methane as that performed for carbon monoxide. The uncertainty in the line strengths was kept at $3.5 \%$ and the RMS noise level was based on the window size; however, the uncertainty in the broadening coefficient was increased to $20 \%$ because the air-broadened values were used. Concentrations from $\sim 0.5$ to 50 Torr were compared to the computed values. The computed results are provided in Table I, and they agree with the manometric mixtures within the estimated uncertainty levels. In general, the agreement between the two is very good-on average $\sim 6 \%$-but not as close as for the carbon monoxide results.

Carbon Dioxide Results. Although carbon dioxide is not produced directly from the Fischer-Tropsch reaction (Eq. 1), a small amount of carbon dioxide is produced via the Boudouard reaction:

$$
2 \mathrm{CO} \rightleftharpoons \mathrm{CO}_{2}+\mathrm{C}
$$

In the reaction in Eq. 14, carbon monoxide disproportionates, producing carbon dioxide and deposited carbon. Carbon dioxide has a set of very strong lines centered at $\sim 2350 \mathrm{~cm}^{-1}$. Similar to carbon monoxide, mixtures of carbon dioxide and air were made and the

Volume 69, Number 11, 2015 
TABLE I. Comparison between prepared mixtures of the FTT gases with hydrogen as the background gas and ?14 values calculated from measured FT-IR spectra.

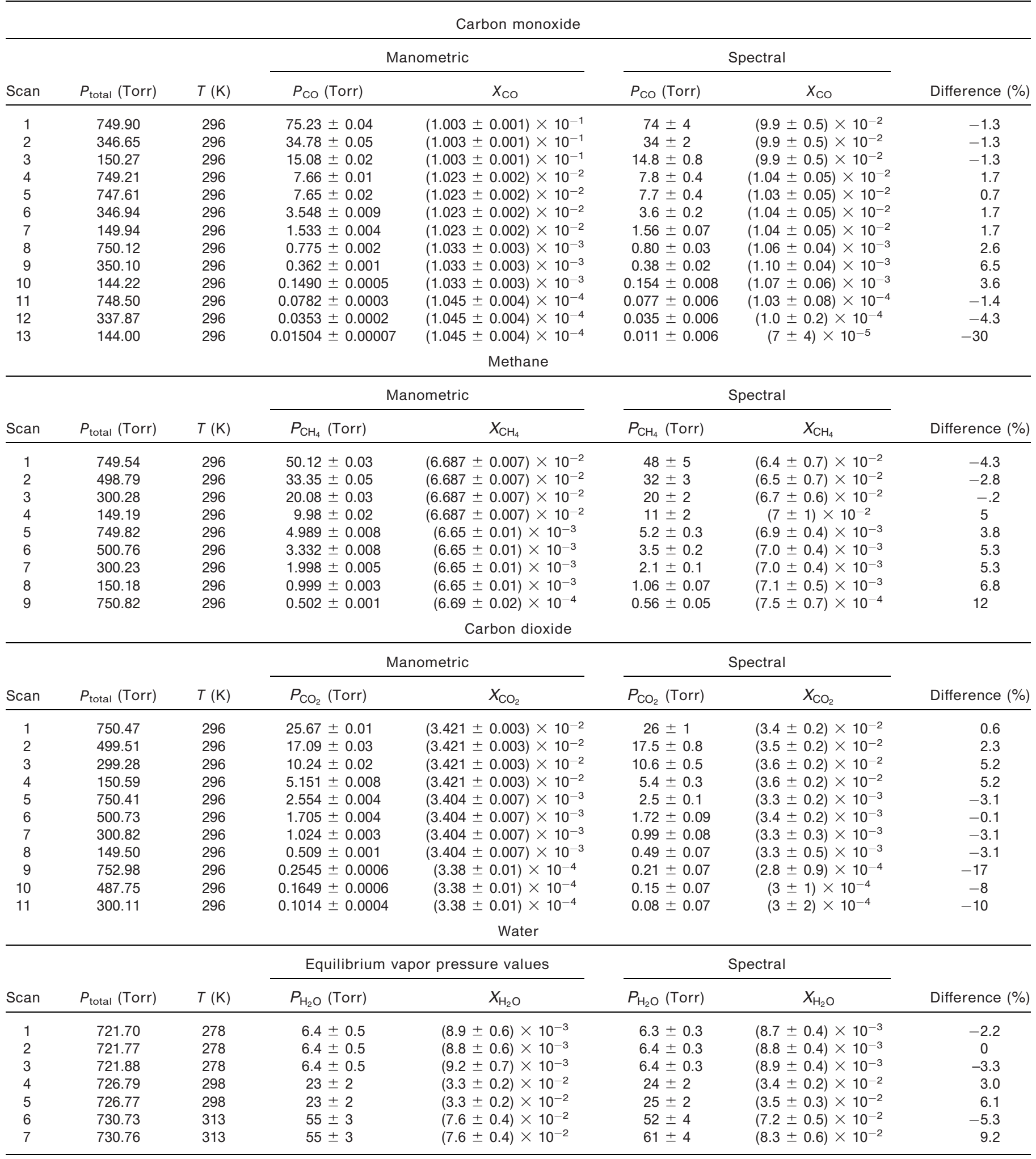

resulting spectra were compared with the calculated spectra with very good results. Unfortunately, with the strong carbon dioxide lines in hydrogen gas, there were noticeable differences in the line shapes between the two sets of spectra. Unlike carbon monoxide, data on the broadening of the carbon dioxide lines in hydrogen were not available. Burch et al. ${ }^{23}$ did a study of the broadening of this band due to hydrogen and reported the foreign gas broadening coefficient as 1.17 . This number represents the average increase in pressure over the nitrogen 


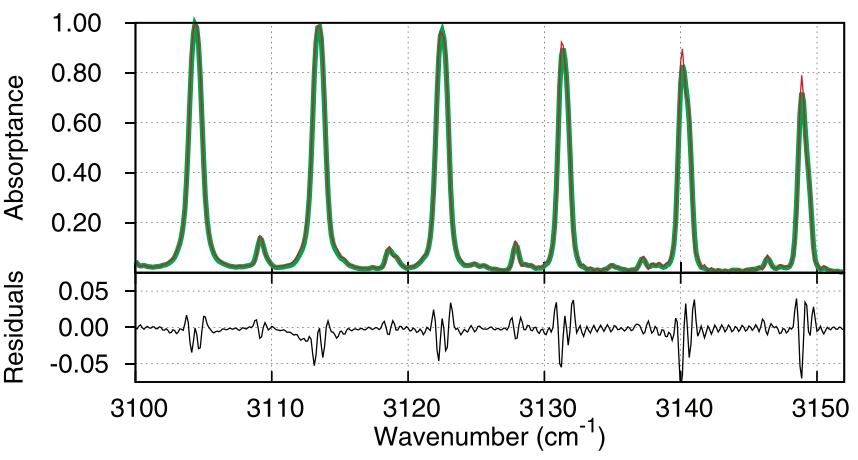

(a)

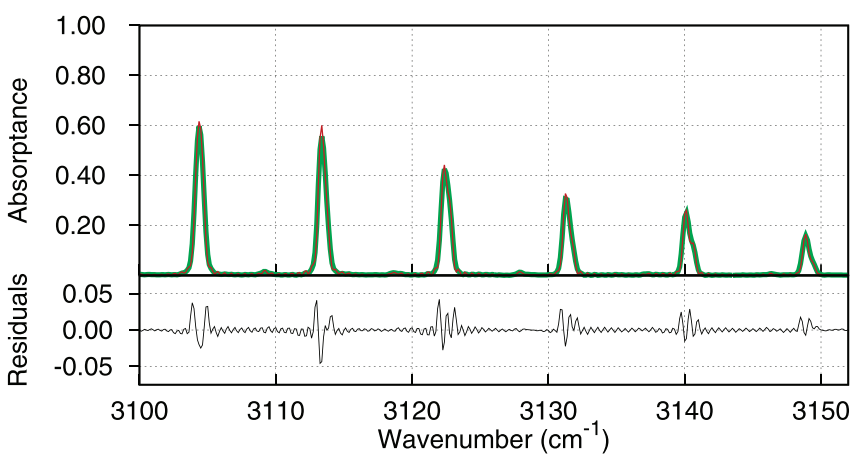

(b)

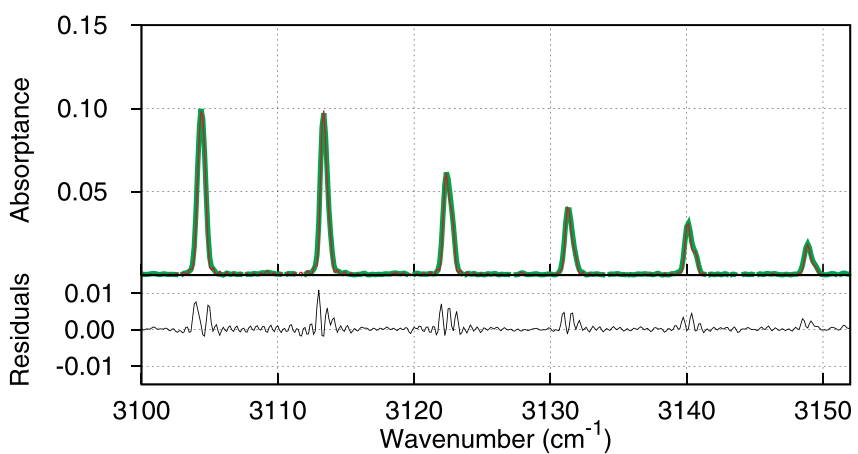

(c)

Experimental Calculated

FIG. 4. Comparison between the measured and calculated spectra for methane $\left(\mathrm{CH}_{4}\right)$ in hydrogen at $296 \mathrm{~K}$ and plots of the residuals between the measured and calculated spectra. (a) For 50.1 Torr $\mathrm{CH}_{4}$ at $749.5 \mathrm{Torr}$ total pressure. (b) For 4.99 Torr $\mathrm{CH}_{4}$ at 749.8 Torr total pressure. (c) For 0.50 Torr $\mathrm{CH}_{4}$ at 750.8 Torr total pressure.

broadening coefficient to give the same absorption over the band. However, it is an average value over the band and gives no information on how particular lines vary. A series of tests were made by simply increasing all airbroadened coefficients by $17 \%$. Although these values produced a much better match to the carbon dioxide in hydrogen spectra, the change was still not close enough to match the line shape over the whole band for the quantitative measurements desired in this work.

As an alternative, a small region of weak carbon dioxide lines were chosen to make the quantitative comparisons. These lines cover the range 2257$2287 \mathrm{~cm}^{-1}$ and are relatively insensitive to the foreign gas broadening coefficient over the experimental concentration range, yet they are still strong enough to give a sufficient signal in this range, $\sim 0.5--25$ Torr.
Figure 5 is a comparison plot between the carbon dioxide spectra measured in an atmosphere of hydrogen and the calculated spectra. These plots include 25.7 Torr carbon dioxide at 750.5 Torr total pressure (Fig. 5a), 2.55 Torr carbon dioxide at 750.4 Torr total pressure (Fig. $5 b)$, and 0.254 Torr carbon dioxide at 753.0 Torr total pressure (Fig. 5c). A plot of the residuals between the measured and calculated spectra is also shown for each pair of spectra. In general, the agreement between the two is again very good, although the signal level in Fig. $5 \mathrm{c}$ shows the level to be very small and very susceptible to the noise level in the measured spectra.

As before, several mixtures of carbon dioxide were generated under atmospheres of hydrogen and carbon dioxide partial pressures were calculated for the measured spectra. A comparison between the manometric mixtures and the concentrations computed from the calculated spectra are given in Table I. The uncertainty levels in the computed values were estimated, as before, from the RMS noise level in a typical spectrum in the spectral range, a $3.5 \%$ uncertainty in the carbon dioxide line strength, and a $20 \%$ uncertainty in the foreign gas broadening coefficient. In general, the agreement in the desired pressure range ( 0.5-25 Torr) is very good, and it is estimated that the calculated uncertainty should be less than $10 \%$ in this range.

Water Results. The last investigated species represents a case, as mentioned earlier, in which experimental calibrations are not easily made and there is an advantage to using the HITRAN database and calculated spectra as an alternative. In this case, there is some difficulty producing a range of water partial pressures to produce the calibration data. Based on the previous good matches with other species in this study, a similar close match is expected. Still, some type of validation of the approach using water is needed.

To compare the measured and calculated spectra for water, the following test was performed. A small quantity of purified water was added to the experimental cell and sealed. The cell was then carefully covered with copper tubing and insulated on the outside. This tubing was attached to the recirculating pump of a RM20 refrigerating bath (Brinkmann Lauda). The cell was then evacuated, purged with hydrogen gas, and filled to approximately 720 Torr hydrogen. The spectra were taken at various recirculating bath temperatures. The water vapor concentration was assumed to be equal to the equilibrium vapor pressure of the water at the cell temperature, as measured using a type-K thermocouple.

Figure 6 shows a comparison between measured and calculated spectra over the range $3800-3910 \mathrm{~cm}^{-1}$ for 55.4 Torr water at 730.7 Torr total pressure (Fig. 6a), 23.4 Torr water at 726.8 Torr total pressure (Fig. 6b), and 6.43 Torr water at 721.8 Torr total pressure (Fig. 6c). As before, below each pair of spectra is a plot of the residuals between the measured and computed spectra. In general, the match between the measured and calculated spectra is very good. Table I shows a comparison between the vapor concentrations assuming equilibrium within the cell and the results computed from the experimentally measured spectra. The uncertainty in the equilibrium vapor pressure value is based on an estimated $\pm 1 \mathrm{~K}$ uncertainty in the measured cell

Volume 69, Number 11, 2015 


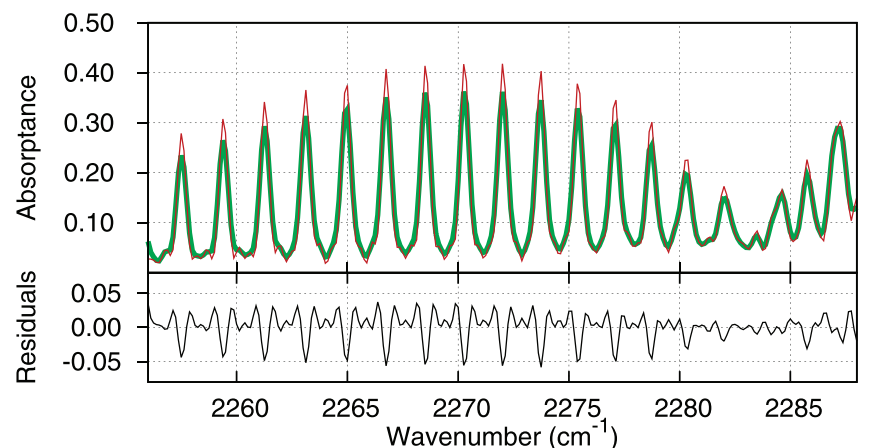

(a)

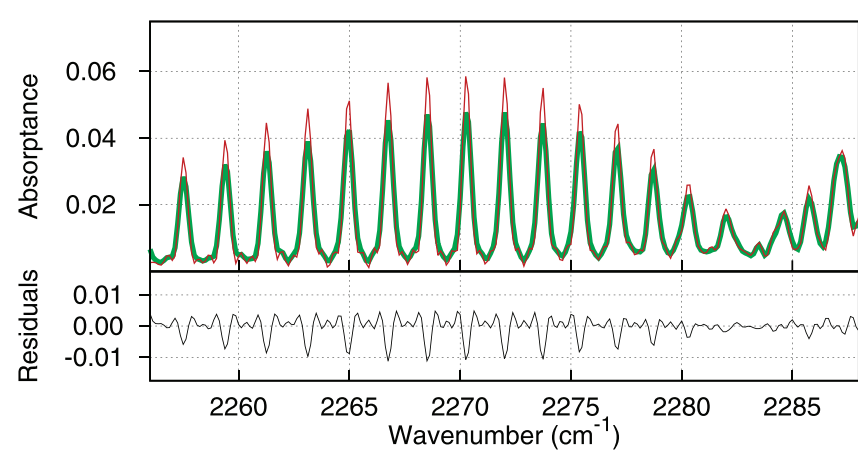

(b)

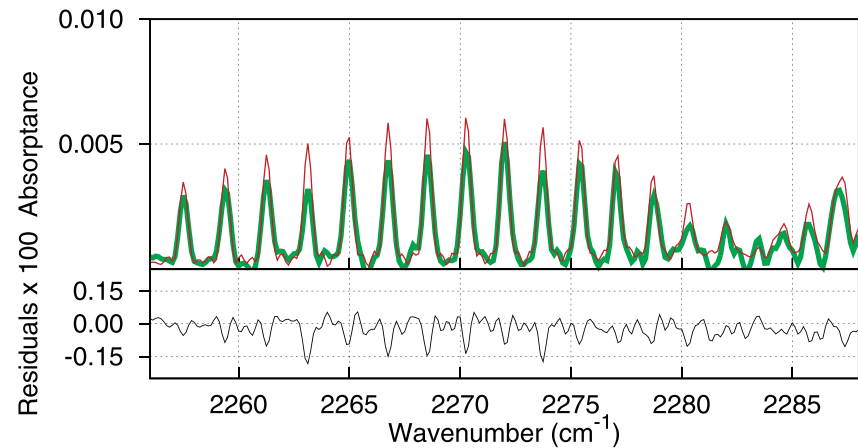

(c)

Experimental Calculated

FIG. 5. Comparison between measured and calculated spectra for carbon dioxide $\left(\mathrm{CO}_{2}\right)$ in hydrogen at $296 \mathrm{~K}$ and plots of the residuals between the measured and calculated spectra. (a) For 25.7 Torr $\mathrm{CO}_{2}$ at 750.5 Torr total pressure. (b) For 2.55 Torr $\mathrm{CO}_{2}$ at 750.4 Torr total pressure. (c) For 0.254 Torr $\mathrm{CO}_{2}$ at 753.0 Torr total pressure.

temperature. The uncertainty in the spectral calculation is taken from the equivalent uncertainties noted previously: a $3.5 \%$ uncertainty in the line strength, a $20 \%$ uncertainty in the air-broadening coefficient, and three times the RMS noise level in the spectral range of the measurement. Not surprisingly, there is a larger fluctuation in the percentage differences between the two measurements. Although most values agree within the stated level of uncertainties, scans 4 and 6 differ by a little more than this range.

\section{SYNTHETIC CALIBRATIONS AND FISCHER- TROPSCH-TYPE EXPERIMENTS}

In the previous sections, very good agreement between various gas mixtures over a range of concen-

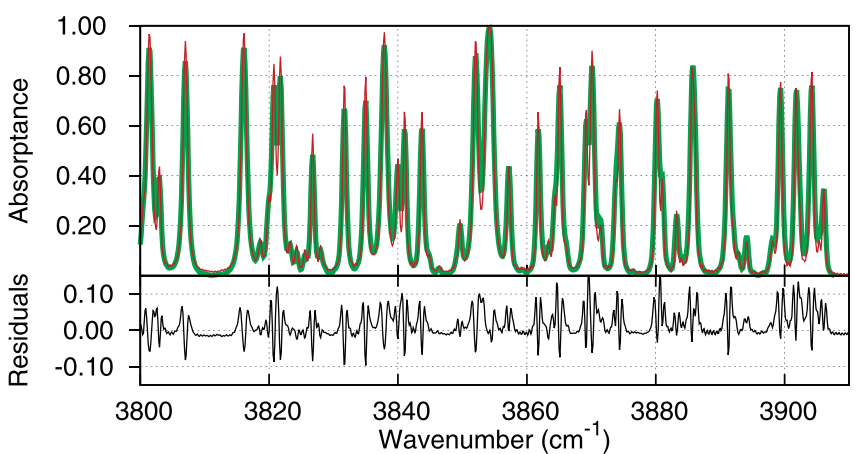

(a)

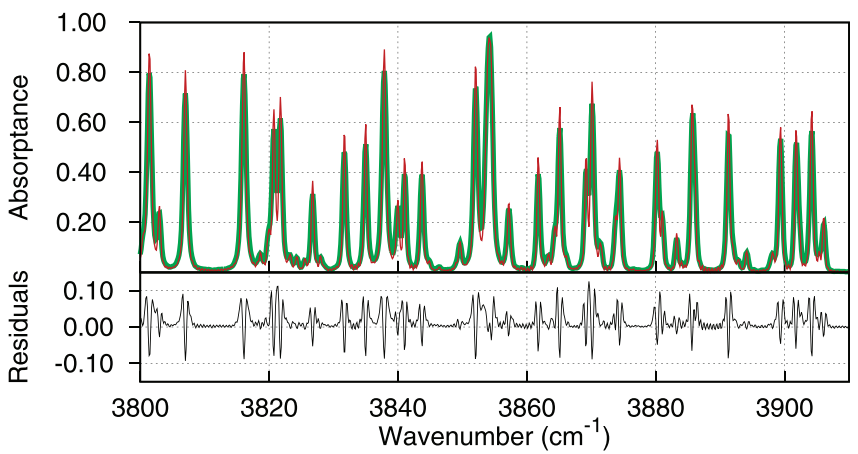

(b)

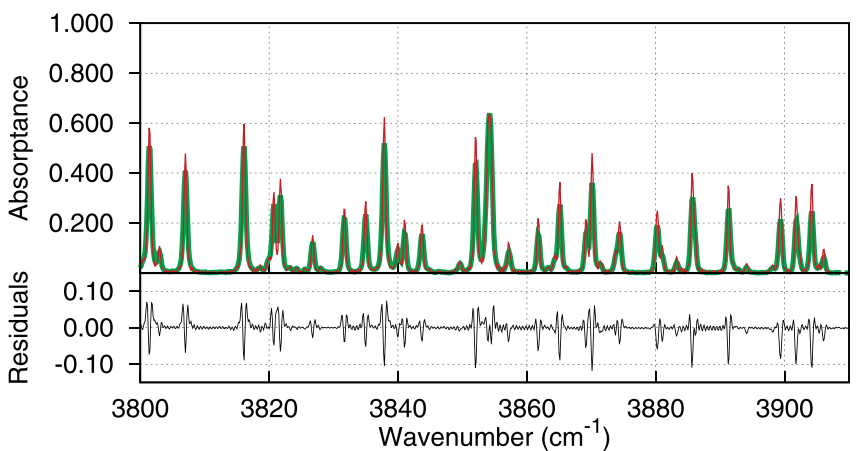

(c)

Experimental Calculated

FIG. 6. Comparison between measured and calculated spectra for water $\left(\mathrm{H}_{2} \mathrm{O}\right)$ in hydrogen and plots of the residuals between the measured and calculated spectra. (a) For 55.4 Torr $\mathrm{H}_{2} \mathrm{O}$ at 730.7 Torr total pressure and $313 \mathrm{~K}$. (b) For 23.4 Torr $\mathrm{H}_{2} \mathrm{O}$ at 726.8 Torr total pressure and $298 \mathrm{~K}$. (c) For 6.43 Torr $\mathrm{H}_{2} \mathrm{O}$ at 721.8 Torr total pressure and $278 \mathrm{~K}$.

trations and values derived from FT-IR spectra has been demonstrated. Although the bisection method is useful in locating the match between areas from computed and measured spectra and could be used to process data from a FTT experiment, such a calculation would be impractical due to the large computational time required. The time required to compute a single high-resolution spectrum can vary from several seconds to, more often, several minutes. Therefore, to locate a single root for a single gas concentration takes several hours. A typical FTT run has $\sim 100$ of these data points, with several different gases, making the bisection method impractical.

An alternative method, based on the good agreement between the calculated values from the HITRAN data- 


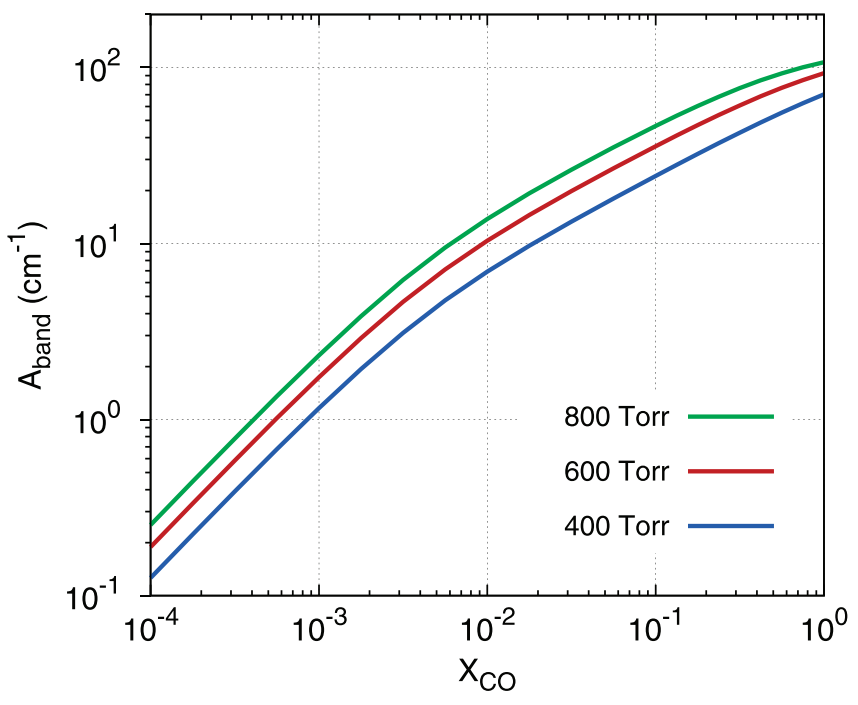

FIG. 7. Example of synthetic calibration data for carbon monoxide. The plot shows the integrated absorptance as a function of carbon monoxide mole fraction over the range $2080-2205 \mathrm{~cm}^{-1}$ at $296 \mathrm{~K}$.

base and the measured concentrations, is to generate these spectra over a range of conditions that should bracket any experimental conditions. These spectra can then be used to build synthetic calibration plots that can be interpolated to give the experimental concentrations. Such calibration plots have the advantage of covering a range of conditions that may not be practical to collect experimentally.

Such a plot is shown for carbon monoxide in Fig. 7. As the FTT reaction proceeds, the total pressure typically changes. For a given total pressure, the spectra are calculated from the HITRAN database and the apodization procedure, and the area over the specific spectral range is calculated. These spectra are calculated for a range of fixed values of the gas mole fraction that brackets the experimental concentration range. In Fig. 7, the area of the band, $A_{\text {band, }}$, is plotted as a function of the mole fraction of carbon monoxide for different total system pressures. For clarity, data for only three total pressures are calculated, but for FTT calculations, many more ranges are used. For a given data point in an FTT run with a specific total pressure, the data of the two curves bracketing this pressure range can be interpolated to give gas mole fractions as a function of band area; the final concentration is calculated from these data.

There are several advantages to computing the gas concentration using such synthetic calibration curves. First, the time-consuming calculation of spectra can be made beforehand and, given enough time, to any degree of precision needed. In addition, these results can be continually improved with new data that can be added to the database later. Second, if the experimental conditions change (e.g., a different cell with a different path length is used or the cell temperature changes), new calibration data can easily be computed. Synthetic calibration data can also be generated if the FT-IR spectrometer details such as resolution or apodization function change.

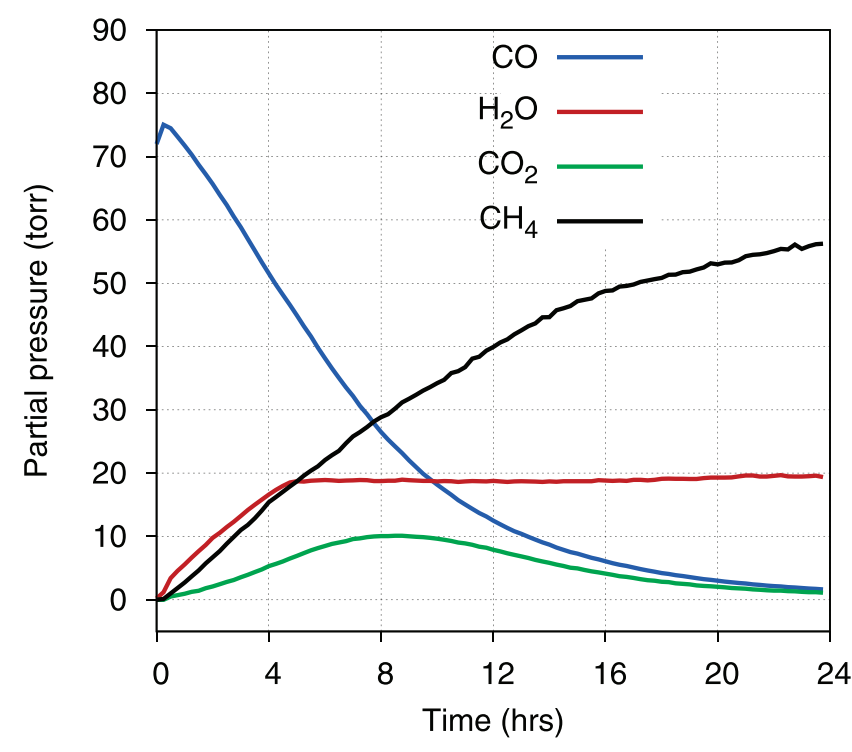

FIG. 8. Example of a FTT catalyst run with partial pressures calculated via synthetic calibration data.

Once the synthetic calibration data are available, the interpolation of these values gives a rapid calculation of the concentration that can be made as the FTT run is in progress. Figure 8 is an example of this process applied to spectra collected from a FTT run using an iron catalyst at $600{ }^{\circ} \mathrm{C}$. Over the course of $24 \mathrm{~h}$, the initial charge of carbon monoxide has been depleted, resulting in 56 Torr methane. Carbon dioxide is formed as an intermediate species, rising to a maximum of $\sim 10$ Torr, and then it too is consumed. Water vapor is produced quickly and rises to a pressure consistent with equilibrium with its liquid phase vapor pressure. Such plots can now be made to quantitatively compare the rates of change of these partial pressure values using different substrate materials or previously used substrates that have been coated with organic material.

\section{SUMMARY AND DISCUSSION OF RESULTS}

The goal of this research is to develop a method of quantifying gas concentrations during Fischer-Tropschtype catalytic reactions. During an experimental run, the gases above such catalysts are recirculated through the cell of an FT-IR spectrometer, which is used to take spectra at timed intervals. Molecular absorption data for many of the species in the FTT reactions are available in the HITRAN database. While the spectra for many of the gas species in the FTT reactions may be calculated, the measured spectra are broadened using the ILS of the spectrometer. A technique in which the same mathematical operations that occur in a spectrometer are applied to a calculated high-resolution spectrum to account for this ILS. The result is a calculated transmission spectrum that is a very close match to the measured spectrum.

The accuracy of this technique was tested by preparing various mixtures of the FTT gases and comparing these with the gas concentrations calculated from the measured spectra. Based on this good agreement, synthetic calibration data were calculated

Volume 69, Number 11, 2015 
for the FTT gases using this technique, allowing the rapid computation of the gas concentrations during an FTT experimental run.

Two difficulties in applying the technique to FTT experiments are (1) that the gases involved in the FTT reaction have rather large partial pressures, and hence strong absorption features, and (2) that the predominant background gas is hydrogen. Because the HITRAN database includes only foreign gas broadening data due to air, the researcher must use care in choosing the integration regions for the quantitative calculations. In the current study, the technique has been applied only to the major, infrared-absorbing FTT species. In previous experimental studies, both ethane and ethylene were detected as minor products in the FTT reactions. Data for both these molecules are available in the HITRAN database, and the current study probably could also have been applied to these species. Finally, in addition to providing a means for comparing different FTT reaction rates, this quantitative analysis may possibly be used to track the amount of carbon contained in the gaseous species. If so, then it may be possible to determine the fraction of the initial carbon that is introduced via the carbon monoxide feedstock in the system that gets deposited on the dust grain surfaces during a FTT reaction.

\section{ACKNOWLEDGMENT}

The authors gratefully acknowledge support for this work from NASA's Exobiology Program.

1. S.L. Miller, H.C. Urey. "Organic Compound Synthesis on the Primitive Earth". Science. 1959. 130(3370): 245-251.

2. J.E. Elsila, D.P. Glavin, J.P. Dworkin. "Cometary Glycine Detected in Samples Returned by Stardust". Meteorit. Planet. Sci. 2009. 44: 1323-1330

3. H.G.M. Hill, J.A. Nuth. "The Catalytic Potential of Cosmic Dust: Implications for Prebiotic Chemistry in the Solar Nebula and Other Protoplanetary Systems". Astrobiology. 2003. 3(2): 291-304.

4. J.A. Nuth III, N.M. Johnson, S. Manning. "A Self-Perpetuating Catalyst for the Production of Complex Organic Molecules in Protostellar Nebulae". Astrophys. J. 2008. 673(2): L225-L228.

5. L.S. Rothman, I.E. Gordon, Y. Babikov, A. Barbe, D.C. Brenner, P.F Bernath, M. Birk, L. Bizzocchi, V. Boudon, L.R. Brown, A Campargue, K. Chance, E.A. Cohen, L.H. Coudert, V.M. Devi, B.J. Drouin, A. Fayt, J.-M. Flaud, R.R. Gamache, J.J. Harrison, J.-M. Hartmann, C. Hill, J.T. Hodges, D. Jacquemart, A. Jolly, J. Lamouroux, R.J. Le Roy, G. Li, D.A. Long, O.M. Lyulin, C.J. Mackie, S.T. Massie, S. Mikhailenko, H.S.P. Müller, O.V. Naumenko, A.V. Nikitin, J. Orphal, V. Perevalov, A. Perrin, E.R. Polovtseva, C Richard, M.A.H. Smith, E. Starikova, K. Sung, S. Tashkun, J. Tennyson, G.C. Toon, V.G. Tyuterev, G. Wagner. "The HITRAN Database." Harvard-Smithsonian Center for Astrophysics. https:// www.cfa.harvard.edu/hitran/ [accessed Mon day year]

6. L.S. Rothman, I.E. Gordon, Y. Babikov, A. Barbe, D.C. Brenner, P.F. Bernath, M. Birk, L. Bizzocchi, V. Boudon, L.R. Brown, A Campargue, K. Chance, E.A. Cohen, L.H. Coudert, V.M. Devi, B.J. Drouin, A. Fayt, J.-M. Flaud, R.R. Gamache, J.J. Harrison, J.-M. Hartmann, C. Hill, J.T. Hodges, D. Jacquemart, A. Jolly, J. Lamouroux, R.J. Le Roy, G. Li, D.A. Long, O.M. Lyulin, C.J. Mackie,
S.T. Massie, S. Mikhailenko, H.S.P. Müller, O.V. Naumenko, A.V. Nikitin, J. Orphal, V. Perevalov, A. Perrin, E.R. Polovtseva, C. Richard, M.A.H. Smith, E. Starikova, K. Sung, S. Tashkun, J. Tennyson, G.C. Toon, V.G. Tyuterev, G. Wagner. "The HITRAN2012 Molecular Spectroscopic Database". J. Quant. Spectrosc. Radiat. Transfer. 2013. 130(4): 4-50.

7. D.W.T. Griffith. "Synthetic Calibration and Quantitative Analysis of Gas-Phase FT-IR Spectra”. Appl. Spectrosc. 1996. 50(1): 59-70.

8. P.W. Morrison, O. Taweechokesupsin. "Calculation of Gas Spectra for Quantitative Fourier Transform Infrared Spectroscopy of Chemical Vapor Deposition”. J. Electrochem. Soc. 1998. 145(9): 3212-3219.

9. P.R. Griffiths, J.A. De Haseth. Fourier Transform Infrared Spectrometry. Hoboken, NJ: John Wiley and Sons, 2007. 2nd ed.

10. N.W. Cant, D.C. Chambers, I.O.Y. Liu. "The Reduction of NO by CO in the Presence of Water Vapour on Supported Platinum Catalysts: Formation of Isocyanic Acid (HNCO) and Ammonia". Appl. Catal., B. 2003. 46: 551-559.

11. E. Flores, J. Viallon, P. Moussay, R.I. Wielgosz. "Accurate Fourier Transform Infrared (FT-IR) Spectroscopy Measurements of Nitrogen Dioxide $\left(\mathrm{NO}_{2}\right)$ and Nitric Acid $\left(\mathrm{HNO}_{3}\right)$ Calibrated with Synthetic Spectra”. Appl. Spectrosc. 2013. 67(10): 1171-1178.

12. R.J. Yokelson, R. Susott, D.E. Ward, J. Reardon, D.W.T. Griffith. "Emissions from Smoldering Combustion of Biomass Measured by Open-Path Fourier Transform Infrared Spectroscopy". J. Geophys. Res. 1997. 102(D15): 18865-18877.

13. A.D. Cowan, N.W. Cant, B.S. Haynes, P.F. Nelson. "The Catalytic Chemistry of Nitromethane over Co-ZSM5 and Other Catalysts in Connection with the Methane-NO SCR Reaction". J. Catal. 1998. 176: 329-343.

14. T.E.L. Smith, M.J. Wooster, M. Tattaris, D.W.T. Griffith. "Absolute Accuracy and Sensitivity Analysis of OP-FTIR Retrievals of $\mathrm{CO}_{2}$, $\mathrm{CH}_{4}$ and $\mathrm{CO}$ over Concentrations Representative of 'Clean Air' and 'Polluted Plumes'”. Atmos. Meas. Tech. 2011. 4: 97-116.

15. I.R. Burling, R.J. Yokelson, D.W.T. Griffith, T.J. Johnson, P. Veres, J.M. Roberts, C. Warneke, S.P. Urbanski, J. Reardon, D.R. Weise, W.M. Hao, J. de Gouw. "Laboratory Measurements of Trace Gas Emissions from Biomass Burning of Fuel Types from the Southeastern and Southwestern United States". Atmos. Chem. Phys. 2010. 10: 11115-11130

16. N.W. Cant, D.E. Angove, D.C. Chambers. "Nitrous Oxide Formation During the Reaction of Simulated Exhaust Streams over Rhodium, Platinum and Palladium Catalysts". Appl. Catal., B. 1998. 17: 63-73.

17. T. Fleckl, H. Jäger, I. Obernberger. "Experimental Verification of Gas Spectra Calculated for High Temperatures Using the HITRAN/ HITEMP Database”. J. Phys. D: Appl. Phys. 2002. 35(23): 3138-3144.

18. A.V. Sepman, R. den Blanken, R. Schepers, L.P.H. de Goey. "Quantitative Fourier Transform Infrared Diagnostics of the GasPhase Composition Using the HITRAN Database and the Equivalent Width of the Spectral Features". Appl. Spectrosc. 2009. 63(11): 12111222.

19. R.H. Norton, R. Beer. "New Apodizing Functions for Fourier Spectrometry". J. Opt. Soc. Am. 1976. 66(3): 259-264.

20. R. den Blanken. Equivalent Width Method for Quantitative FTIR Analysis of Biomass Pyrolysis Using HITRAN Database. [Ph.D. Dissertation]. Eindhoven, Netherlands: Technische Universiteit Eindhoven, 2008.

21. L. Régalia-Jarlot, X. Thomas, P. Von der Heyden, A. Barbe. "Pressure-Broadened Line Widths and Pressure-Induced Line Shifts Coefficients of the (1-0) and (2-0) Bands of ${ }^{12} \mathrm{C}^{16} 0$ ". J. Quant. Spectrosc. Radiat. Trans. 2004. 91(2): 121-131.

22. S.P. Fuss, M.J. Hall, O.A. Ezekoye. "Band-Integrated Infrared Absorptance of Low-Molecular Weight Paraffin Hydrocarbons at High Temperatures”. Appl. Opt. 1999. 38(13): 2895-2904.

23. D.E. Burch, E.B. Singleton, D. Williams. "Absorption Line Broadening in the Infrared". Appl. Opt. 1962. 1(3): 359-363. 
1. Author: This article has been converted from LaTeX to Word and edited for grammar, style, and usage. Please pay particular attention to the equations as they have been formatted according to journal style (e.g., Greek letters are never italic, etc.). Please compare it with your original document and make corrections on these pages. Please limit your corrections to substantive changes that affect meaning. If no change is required in response to a question, please respond "OK as set." Copyeditor

2. Author: Please verify author names and addresses. Copyeditor

3. AU: In sentence beginning, "After collecting the samples..." please verify; the reaction vessel is not labeled in Fig. 1. Copyeditor

4. AU: In the sentence beginning, "The temperature of the mantle was ..." please provide manufacturers. Copyeditor

5. Author: In the sentence beginning "The reactant gases were..." please provide manufacturer. Copyeditor

6. AU: In sentence beginning "Second, it transported the gases..." please provide manufacturer. Copyeditor

7. Author: The sentence, "This technique is well suited..." was rewritten because "expected" was used twice. Please verify I have maintained your meaning here. Editor

8. Author: In the sentence beginning, "The absorption coefficient. .." please verify that this change is correct; $v_{c, i}$ is identified as the transition frequency later. Copyeditor

9. AU: In the sentence beginning "For other temperatures..." please verify that $v_{c, i}$ is correct. Copyeditor

10. Author: The phrase "As noted in the Introduction..." was removed from the sentence that now begins as, "As noted earlier in this work, the measured transmission..." because we could not find this discussion in the Introduction section. Please confirm. Copyeditor and Editors

11. Author: In the sentence beginning, "Régalia-Jarlot et al. provided. . ." please verify citation number is correct here and throughout.

12. Author: In the sentence beginning "The cell was then carefully covered..." please provide manufacturer. Copyeditor

13. Author: Please verify the list of authors and provide a date in which this URL was initially accessed. Copyeditor

14. Author: This table was changed to conform to journal format and style. Please verify all data in the event something was missed in the conversion. Copyeditor 